\title{
Hashimoto's Encephalopathy Presenting With Altered Mental Status and Myoclonus and Co-Occurring With Psoriasis
}

\author{
Arshi Naz ${ }^{\mathrm{a}, \mathrm{c}}$, Muhammad M Sheikh ${ }^{\mathrm{b}}$, Mayada Issa ${ }^{\mathrm{b}}$
}

\begin{abstract}
We report the case of Hashimoto's encephalopathy (HE) in a 48-year-old male who presented with sudden onset of unresponsiveness with reported confusion and behavioral changes 3 days prior. Metabolic derangement, infectious and toxic factors were ruled out through laboratory testing. Stroke was excluded through brain CT and MRI. Vasculitis panel was unremarkable. Extensive CSF study was unremarkable except for high protein consistent with similar finding in other reported cases of HE. His TSH was normal but his anti-thyroid peroxidase antibody was highly elevated indicative of HE. EEG showed diffuse slow wave pattern. 14-3-3 protein, which is a non-specific marker for Creutzfeldt-Jakob disease, was not detected. The patient showed marked improvement with corticosteroid treatment and his mental status returned to baseline. Our patient also has a history of psoriasis. While HE is reported to be associated with many autoimmune disorders, the currently reported co-occurrence with psoriasis is rare and this report, to the best of our knowledge, is only the second such report. We emphasize that HE should be considered as a differential diagnosis in patients with altered mental status when metabolic, infectious and toxic factors have been ruled out, early onset dementias can be ruled out, and presence of other autoimmune disorders are noted. Anti-thyroid peroxidase (anti-TPO) antibody, although being a non-specific marker, should be checked in such cases.
\end{abstract}

Keywords: Hashimoto's encephalopathy; Autoimmune; Antithyroid peroxidase; Creutzfeldt-Jakob disease; Dementia; Altered mental status; Myoclonus; Behavior changes

\footnotetext{
Manuscript accepted for publication February 21, 2013

${ }^{a}$ Department of Neurology, West Virginia University, 1 Medical Center Drive, Morgantown, WV 26505, USA

${ }^{\mathrm{b}}$ Department of Medicine, West Virginia University, 1 Medical Center Drive, Morgantown, WV 26505, USA

${ }^{\mathrm{c}}$ Corresponding author: Arshi Naz, Department of Neurology, West Virginia University, 1 Medical Center Drive, Morgantown, WV 26505, USA. Email: arshinaz@gmail.com
}

doi: http://dx.doi.org/10.4021/jmc1144w

\section{Introduction}

Hashimoto's encephalopathy (HE) is a rare neuroendocrine disorder associated with Hashimoto's thyroiditis. Also referred to as steroid-responsive encephalopathy associated with autoimmune thyroiditis (SREAT), the condition is responsive to treatment with corticosteroids but subsequent relapse is often reported. Clinical symptoms vary from altered mental status including cognitive dysfunction and behavioral changes, seizures, stroke-like symptoms, myoclonus, ataxia, gait disturbance, psychosis, mood disturbance, sleep disorders and others. High titer of anti-thyroid peroxidase (antiTPO) antibody is often reported in patients. We report the case of a 48-year-old male who presented with sudden onset unresponsiveness, altered mental status, myoclonus and sleep disturbances and laboratory findings consistent with HE.

\section{Case Report}

A 48-year-old male with no significant past medical history other than psoriasis was brought to our facility after being found by family members standing firmly against a wall, with clenched teeth, wide open eyes and flexed arms. He was unresponsive and remained in the same posture for an hour before he was brought to the emergency room. No tonic-clonic activities or incontinence were noted by the family. This episode was preceded by what family described as odd behavior, confusion and increased anxiety for 3 days. No known similar episodes in the past were reported. Mild insomnia was also reported.

He was admitted to Medical intensive care unit and intubated for airways protection. On physical exam, patient was somnolent but he opened his eyes to tactile stimulation. $\mathrm{He}$ was not responsive to verbal stimulation and was not following commands. Patient was noted to have mild tremors. Patient was moving all his extremities spontaneously. Pupils were equal and round, reactive to light and accommodation and corneal reflexes were intact. No facial asymmetry was noted. His tongue had bite marks on the right side but no 
fasciculation or atrophy. He had increased tone in his upper extremities. Patient was given Midazolam for concern of seizure and Naloxone for suspected drug overdose. Naloxone did not improve his mental status.

We performed a number of tests to rule out metabolic, infectious and toxic causes of his altered mental status and sudden unresponsiveness. His basic metabolic panel was normal. Ammonia, vitamin B12 and folic acid levels were normal. Thyroid stimulating hormone (TSH) level was normal (4.417 $\mu \mathrm{IU} / \mathrm{mL}$, Reference: 0.3 - $5.9 \mu \mathrm{IU} / \mathrm{mL})$. Erythrocyte sedimentation rate (ESR) was normal $(3 \mathrm{~mm} / \mathrm{hr}$, Reference: 0 - $15 \mathrm{~mm} / \mathrm{hr}$ ) but C-reactive protein (CRP) was elevated (3.026 mg/dL, Reference: $<1.0 \mathrm{mg} / \mathrm{dL})$. Alanine Aminotransferase (ALT) was normal (14 U/L, Reference: 7 - $55 \mathrm{U} / \mathrm{L})$. Aspartate Aminotransferase (AST) was normal as well (21 U/L, Reference: 8 - $48 \mathrm{U} / \mathrm{L})$.

Lumbar puncture was performed and cerebrospinal fluid (CSF) study showed nucleated cell count of 2 , protein 154 $\mathrm{mg} / \mathrm{dL}$ (Reference: 15 - $45 \mathrm{mg} / \mathrm{dL}$ ) and glucose $81 \mathrm{mg} / \mathrm{dL}$ (Reference: 40 - $70 \mathrm{mg} / \mathrm{dL}$ ). CSF culture with gram stain was negative for any growth. CSF study for Herpes simpex virus, Arbovirus antibody panel and Cryptococcal antigen were negative. Fungus culture in CSF was negative as well. Tick-borne serology was negative. Blood culture for bacterial and fungal infections were also negative. HIV screening was negative. QuantiFERON In-Tube blood test for Mycobacterium Tuberculosis antigen was negative. Toxic screening was performed and it was positive for cannabinoid. According to the family, patient used marijuana occasionally.

In order to rule out any acute process going on in the brain, several imaging studies were performed. Computed tomography (CT) scan of the brain did not reveal any acute intracranial process and showed that normal grey white differentiation was preserved. Magnetic resonance imaging (MRI) of the brain was unremarkable as well without evidence of infarcts or intracranial mass lesions. Intra and extra-cranial CT angiogram did not show any large vessel dissection, thrombus or aneurysms. Transthoracic echocardiogram (TTE) was performed to check for septal and valvular defects and vegetation. Ejection fraction was normal with normal systolic and diastolic functions. Vasculitis panel was negative as well. Electroencephalography (EEG) showed severe generalized slowing in the background indicative of non-specific encephalopathy.

Patient regained responsiveness after two days after which he was extubated. He had no apparent deficits in his motor strength, sensation, cranial nerves, coordination and gait. Patient however, remained confused and intermittently agitated. At times, he was extremely aggressive and required as many as 6 people to hold him down. Intermittent mild twitching was noticed in his upper extremities.

After ruling out potential causes of his symptoms, we considered the differential diagnoses of $\mathrm{HE}$ and requested anti-thyroid peroxidase antibody titer. Since patient's symptoms were stable and not deteriorating over time, Creutzfeldt-Jakob disease (CJD) appeared less likely. Patient however, was known to consume hunted deer though he denied consuming brain. CSF study to detect $14-3-3$ protein was performed. The patient was stable in the meantime; he was oriented to place, person and time, yet seemed distanced and answered questions slowly. He was discharged to be followed up as out-patient following the request of the family. In the meantime, anti-thyroid peroxidase antibody titer was found to be significantly elevated ( $>900 \mathrm{IU} / \mathrm{mL}$, Reference: $<9.0 \mathrm{IU} / \mathrm{mL}$ ) and 14-3-3 protein was not detected in the CSF study. The patient was admitted again. During the time patient was out of the hospital, his altered mental status did not improve. He continued to show confusion, odd behavior and periods of aggression but he did not have any episodes of unresponsiveness. On readmission, his TSH was tested again and was found to be normal $(4.9 \mu \mathrm{IU} / \mathrm{mL}$, Reference: $0.3-5.9 \mu \mathrm{IU} / \mathrm{mL})$. CSF study was repeated to rule out the possibility of previous CSF study being carried out in the latent phase of the infection. CSF study again showed elevated protein at $220 \mathrm{mg} / \mathrm{dL}$ - a significant increase over the previous measurement.

He was started on methylprednisolone 1,000 mg intravenous per day for 3 days. He was discharged and sent home on $60 \mathrm{mg}$ per day dosage of prednisone tapering by $10 \mathrm{mg}$ per week for 6 weeks. Patient's condition improved significantly during the course of steroid treatment and according to his family, he returned to his baseline mental status. He was also able to get back to his day-to-day activities.

\section{Discussion}

First reported in 1966 [1], HE remains a poorly understood disorder as connections between the clinical presentation, thyroid disease, auto-antibody patterns and pathogenesis remain obscure. The disorder has been reported in pediatric, adult as well as elderly subjects. In the adult population, the disorder disproportionately affects female population whereas no such bias is seen in pediatric patients. Many case reports and reviews have been dedicated to characterize this disorder further [2-10].

$\mathrm{HE}$ is known to present with varied clinical presentation. Clinical symptoms vary from altered mental status including cognitive dysfunction and behavioral changes, seizures, stroke-like symptoms, myoclonus, psychosis, mood disturbance, sleep disorders and others. Consistent with other reports [3, 6, 9], our patient displayed cognitive dysfunction and behavioral changes. Similar to other reports [3, 9], myoclonus and tremor was also noticed in our patient. Mild insomnia was also displayed by our patient.

Clinical testing has not shown a consistent pattern for HE. Some reported cases have shown elevated serum inflammatory markers such as ESR, CRP and liver transferase en- 
zyme levels [3, 9]. Our patient had a normal ESR and serum aminotransferase levels but his CRP was elevated. HE is often characterized by detection of elevated titers of anti-TPO antibodies $[3,5,7,9,10]$. While anti-TPO antibodies are still not considered specific marker for HE, it is found in almost $100 \%$ of case reports. Our patient also showed significantly elevated titers of anti-TPO antibodies. No known correlation is available between anti-TPO antibody titer level and severity of the disease. Other antithyroid antibodies such as anti-thyroglobulin (anti-TG) antibodies are also reported in about $50-70 \%$ of reported HE cases $[3,5,11]$.

Patient diagnosed with HE have varied thyroid function level. Majority of cases of HE reported thyroid function of either euthyroid or subclinically hypothyroid $[5,7,9]$. Cases of subclinical and clinical hyperthyroidism with HE are sporadically reported $[5,12]$. The patient reported here showed normal thyroid function and he did not have any history of thyroid dysfunction.

CSF study is commonly done in patients with symptoms pointing to encephalopathy. Majority of cases of HE report elevated CSF protein [3, 5, 7-11]. Our patient had elevated CSF protein which also showed an increasing trend over a 3 week period.

The clinical symptoms of HE and CJD are very similar with dementia, myoclonus, ataxia, personality changes or psychotic symptoms occurring commonly. Commonality of symptoms makes the differentiation between these disorders challenging [11]. CJD, however, is much more rapidly progressing with death occurring in a few months after onset of symptoms. On the other hand, HE patients show significant improvement after therapy with corticosteroids. Although 14-3-3 protein is a non-specific marker for CJD [13], it is commonly checked to get an indication of the cause of encephalopathy. Elevated 14-3-3 protein has also been reported in patients with HE $[14,15]$, thus limiting it's diagnostic significance. 14-3-3 protein was not detected in our patient reported here.

Our patient also displayed slow wave abnormalities in EEG indicative of non-specific encephalopathy. This is consistent with non-specific EEG findings in other reports of HE $[3,5,7-10,16]$. Although the initial presentation indicated possible seizure, EEG ruled out any seizure-like-activity in our patient. Seizure is a common feature of HE. Generalized tonic-clonic, myoclonic, status epilepticus and complex partial seizures have been reported in patients with HE [3, 5-7, $9,10,17,18]$. Our patient did not have any known episode of seizure-like-activity although tongue bites and clenching of jaws were noticed as his initial symptoms.

Brain CT and MRI of our patient were found to be unremarkable. Abnormal findings in brain CT scan of patients with HE have been reported $[5,6]$, however these findings are usually non-specific. The abnormalities may include generalized atrophy and periventricular white matter changes. Abnormal findings in brain MRI of HE patients have also been reported [3, 19] but these findings are also usually nonspecific. The abnormalities may include a diffuse increased signal on T2-weighted and fluid-attenuated inversion recovery (FLAIR) images in the cerebral white matter or dural enhancements.

HE has been reported to co-occur with other autoimmune disorders. In their study, Castillo et al [3] reported patients with HE who had co-morbid autoimmune disorders such as diabetes mellitus type 1, systemic lupus erythematosus, Crohn's disease, sicca syndrome, Sjogren's syndrome and pernicious anemia. HE has also been reported to co-occur with sarcoidosis [20] and psoriatic arthritis [21]. Our patient has a history of psoriasis. The co-occurrence with psoriasis is rare and this case, to the best of our knowledge, is only the second such report of association between HE and psoriasis.

Most cases of HE respond to treatment with high dosage of corticosteroids [5, 7]. In fact, corticosteroid responsiveness has been used as one of the defining criteria for the diagnosis of HE [3]. There is no set duration for the corticosteroid treatment and patient's response to the treatment need to be assessed in deciding the duration. Clinical improvements can be seen in 4 - 6 weeks after high dosage corticosteroid treatment [9] after which the dose can be tapered gradually. Some cases reported to have required prolonged corticosteroid treatment of over 1 year $[3,6,7]$.

\section{Conclusions}

$\mathrm{HE}$ is a rare condition and it is often misdiagnosed. We emphasize, through this case report, that HE should be considered in patients with altered mental status when metabolic, infectious and toxic factors have been ruled out, early onset dementias can be ruled out, and presence of other autoimmune disorders are noted. Laboratory findings such as high protein in CSF study and elevated anti-thyroid peroxidase antibody titers should be considered indicative of HE. The association between $\mathrm{HE}$ and psoriasis, as highlighted in this case report, should also be noted and explored in medical research on HE.

\section{Disclosure}

No relevant financial affiliations or conflicts of interest to disclose.

\section{References}

1. Brain L, Jellinek EH, Ball K. Hashimoto's disease and encephalopathy. Lancet. 1966;2(7462):512-514.

2. Mocellin R, Walterfang M, Velakoulis D. Hashimoto's encephalopathy : epidemiology, pathogenesis and management. CNS Drugs. 2007;21(10):799-811. 
3. Castillo P, Woodruff B, Caselli R, Vernino S, Lucchinetti C, Swanson J, Noseworthy J, et al. Steroid-responsive encephalopathy associated with autoimmune thyroiditis. Arch Neurol. 2006;63(2):197-202.

4. Fatourechi V. Hashimoto's encephalopathy: myth or reality? An endocrinologist's perspective. Best Pract Res Clin Endocrinol Metab. 2005;19(1):53-66.

5. Chong JY, Rowland LP, Utiger RD. Hashimoto encephalopathy: syndrome or myth? Arch Neurol. 2003;60(2):164-171.

6. Chaudhuri A, Behan PO. The clinical spectrum, diagnosis, pathogenesis and treatment of Hashimoto's encephalopathy (recurrent acute disseminated encephalomyelitis). Curr Med Chem. 2003;10(19):1945-1953.

7. Sawka AM, Fatourechi V, Boeve BF, Mokri B. Rarity of encephalopathy associated with autoimmune thyroiditis: a case series from Mayo Clinic from 1950 to 1996. Thyroid. 2002;12(5):393-398.

8. Canton A, de Fabregas O, Tintore M, Mesa J, Codina A, Simo R. Encephalopathy associated to autoimmune thyroid disease: a more appropriate term for an underestimated condition? J Neurol Sci. 2000;176(1):65-69.

9. Kothbauer-Margreiter I, Sturzenegger M, Komor J, Baumgartner R, Hess CW. Encephalopathy associated with Hashimoto thyroiditis: diagnosis and treatment. J Neurol. 1996;243(8):585-593.

10. Shaw PJ, Walls TJ, Newman PK, Cleland PG, Cartlidge NE. Hashimoto's encephalopathy: a steroid-responsive disorder associated with high anti-thyroid antibody titers--report of 5 cases. Neurology. 1991;41(2 ( Pt 1)):228-233.

11. Seipelt M, Zerr I, Nau R, Mollenhauer B, Kropp S, Steinhoff BJ, Wilhelm-Gossling C, et al. Hashimoto's encephalitis as a differential diagnosis of CreutzfeldtJakob disease. J Neurol Neurosurg Psychiatry. 1999;66(2):172-176.

12. Barker R, Zajicek J, Wilkinson I. Thyrotoxic Hashi- moto's encephalopathy. J Neurol Neurosurg Psychiatry. 1996;60(2):234.

13. Shiga Y, Wakabayashi H, Miyazawa K, Kido H, Itoyama Y. 14-3-3 protein levels and isoform patterns in the cerebrospinal fluid of Creutzfeldt-Jakob disease patients in the progressive and terminal stages. J Clin Neurosci. 2006;13(6):661-665.

14. Hernandez Echebarria LE, Saiz A, Graus F, Tejada J, Garcia JM, Clavera B, Fernandez F. Detection of 14-33 protein in the CSF of a patient with Hashimoto's encephalopathy. Neurology. 2000;54(7):1539-1540.

15. Vander T, Hallevy C, Alsaed I, Valdman S, Ifergane G, Wirguin I. 14-3-3 protein in the CSF of a patient with Hashimoto's encephalopathy. J Neurol. 2004;251(10):1273-1274.

16. Schauble B, Castillo PR, Boeve BF, Westmoreland BF. EEG findings in steroid-responsive encephalopathy associated with autoimmune thyroiditis. Clin Neurophysiol. 2003;114(1):32-37.

17. Ferlazzo E, Raffaele M, Mazzu I, Pisani F. Recurrent status epilepticus as the main feature of Hashimoto's encephalopathy. Epilepsy Behav. 2006;8(1):328-330.

18. McGinley J, McCabe DJ, Fraser A, Casey E, Ryan T, Murphy R. Hashimoto's encephalopathy; an unusual cause of status epilepticus. Ir Med J. 2000;93(4):118.

19. Song YM, Seo DW, Chang GY. MR findings in Hashimoto encephalopathy. AJNR Am J Neuroradiol. 2004;25(5):807-808.

20. Archambeaud F, Galinat S, Regouby Y, Magy L, Rebeyrotte I, Vallat JM, Teissier MP. [Hashimoto encephalopathy. Analysis of four case reports]. Rev Med Interne. 2001;22(7):653-659.

21. Wilhelm-Gossling C, Weckbecker K, Brabant EG, Dengler R. [Autoimmune encephalopathy in Hashimoto's thyroiditis. A differential diagnosis in progressive dementia syndrome]. Dtsch Med Wochenschr. 1998;123(10):279-284. 